University of Nebraska - Lincoln

DigitalCommons@University of Nebraska - Lincoln

Faculty Publications from the Department of Engineering Mechanics

Mechanical \& Materials Engineering,

Department of

2010

\title{
Crack nucleation in a peridynamic solid
}

\author{
S. A. Silling \\ Sandia National Laboratories, Albuquerque, NM, sasilli@sandia.gov \\ O. Weckner \\ The Boeing Company, Seattle, WA \\ E. Askari \\ The Boeing Company, Seattle, WA \\ Florin Bobaru Ph.D. \\ University of Nebraska at Lincoln, fbobaru2@unl.edu
}

Follow this and additional works at: https://digitalcommons.unl.edu/engineeringmechanicsfacpub

Part of the Mechanical Engineering Commons

Silling, S. A.; Weckner, O.; Askari, E.; and Bobaru, Florin Ph.D., "Crack nucleation in a peridynamic solid" (2010). Faculty Publications from the Department of Engineering Mechanics. 69.

https://digitalcommons.unl.edu/engineeringmechanicsfacpub/69

This Article is brought to you for free and open access by the Mechanical \& Materials Engineering, Department of at DigitalCommons@University of Nebraska - Lincoln. It has been accepted for inclusion in Faculty Publications from the Department of Engineering Mechanics by an authorized administrator of DigitalCommons@University of Nebraska - Lincoln. 


\title{
Crack nucleation in a peridynamic solid
}

\author{
S. A. Silling • O. Weckner • E. Askari • \\ F. Bobaru
}

Received: 3 August 2009 / Accepted: 5 January 2010 / Published online: 28 January 2010

(C) Springer Science+Business Media B.V. 2010

\begin{abstract}
A condition for the emergence of a discontinuity in an elastic peridynamic body is proposed, resulting in a material stability condition for crack nucleation. The condition is derived by determining whether a small discontinuity in displacement, superposed on a possibly large deformation, grows over time. Stability is shown to be determined by the sign of the eigenvalues of a tensor field that depends only on the linearized material properties. This condition for nucleation of a discontinuity in displacement can be interpreted in terms of the dynamic stability of plane waves with very short wavelength. A numerical example illustrates that cracks in a peridynamic body form spontaneously as the body is loaded.
\end{abstract}

Keywords Crack nucleation - Material stability · Peridynamic $\cdot$ Elasticity

Mathematics Subject Classification (2000) 74B15

S. A. Silling $(\varangle)$

Sandia National Laboratories, Albuquerque, New Mexico, USA

e-mail: sasilli@sandia.gov

O. Weckner · E. Askari

The Boeing Company, Seattle, WA, USA

F. Bobaru

University of Nebraska - Lincoln, Lincoln, NE, USA

\section{Introduction}

Common experience tells us that if a brittle solid body is loaded sufficiently, eventually it fails due to cracking. This paper addresses the following question: How does a crack appear spontanteously in a continuous body? For purposes of this discussion, this spontaneous appearance of such a discontinuity will be called the "nucleation" of a crack.

The field of fracture mechanics is primarily concerned with the evolution of pre-existing defects within a body, rather than the nucleation of new defects. It is frequently noted that real materials, now matter how perfect they seem at the macroscale, invariably contain many defects at the microscale. Therefore, it could be claimed that the nucleation of a macroscale crack never really occurs; instead the seemingly sudden appearance of a discontinuity is merely the growth and coalescence of a large number of pre-existing defects at the smaller scale. However, as a practical matter, an engineering model would require an impractically detailed spatial resolution to capture such a distribution of tiny defects, even if the fundamental mechanics of these defects were adequately understood. So, it is desirable to have a practical mathematical model, applicable at the macroscale, that reproduces the spontaneous emergence of discontinuities.

The classical theory of solid mechanics, because of the requirements for smoothness of the deformation that are built into the fundamental assumptions of the theory, cannot be considered an ideal tool for 
the study of this problem. However, the peridynamic theory (Silling 2000; Silling et al. 2007) has been proposed as a reformulation of solid mechanics whose basic equations remain applicable regardless of the possible emergence of discontinuities in the deformation. In this paper, the nucleation of discontinuities is studied within the peridynamic theory from the point of view of material stability.

Material stability conditions have been investigated within the classical theory as possible criteria for nucleation of discontinuities. For hyperelastic materials in the classical theory, loss of ordinary ellipticity (Knowles and Sternberg 1975, 1978) provides a condition for the presence of static discontinuities in the first spatial derivatives of displacement in a homogeneous body. Material stability conditions with a structure similar to loss of ordinary ellipticity have also been considered as criteria for localization in elastic-plastic materials, particularly for shear bands (Hill 1962; Rudnicki and Rice 1975; Leroy and Ortiz 1989). Klein and Gao (1998) consider a criterion similar to loss of ordinary ellipticity as a condition for crack nucleation. Klein and Gao base this condition on the acoustic tensor corresponding to a material whose response is local but is derived from the response of a network of virtual independent bonds (VIB).

Bazant and Belytschko (1985) conclude that if loss of hyperbolicity (the analogue in the dynamic case of loss of ellipticity in the static case) occurs, then deformations can localize to a discontinuity. However, Belytschko et al. (2003), while adopting this condition as an indicator of crack growth, correctly include the following note of caution: "It must be stressed that these are empirical inferences and that little appears to be known about the morphology and evolution of such surfaces in multi-dimensions."

Although loss of ellipticity has been investigated by many authors as a localization criterion, the mechanical argument connecting it with nucleation of strong discontinuities such as cracks is indirect at best. The reason is that when a discontinuity in displacement forms spontaneously, it is difficult to argue that the fourth order elasticity tensor provides an adequate description of how material on one crack face interacts with material on the other face. This interaction is precisely what should, in a properly formulated mathematical model of a continuum, determine whether a discontinuity grows or does not grow. The ability to model this type of interaction explicitly is one feature that the peri- dynamic theory offers for purposes of modeling crack nucleation. In this paper, a material instability condition is derived that corresponds to the ability of a discontinuous perturbation in the deformation to grow in amplitude over time. The condition involves only the incremental material properties at each point.

Propagation, as opposed to nucleation, of cracks within the peridynamic model has been described elsewhere (Silling and Askari 2005) and has been applied to a number of problems involving complex patterns of fracture (Silling 2003; Silling and Bobaru 2005; Askari et al. 2006; Bobaru 2007; Xu et al. 2007; Colavito et al. 2007; Gerstle et al. 2007; Xu et al. 2008; Agwai et al. 2008a,b; Kilic and Madenci 2009; Kilic et al. 2009; Agwai et al. 2009). Crack growth occurs through the irreversible breakage of bonds as they stretch in the vicinity of a crack tip. The damage condition that governs bond breakage is treated as part of the constitutive model. As shown in Silling and Askari (2005), if bond breakage occurs at a prescribed value of bond stretch, then cracks advance according to the Griffith criterion, consuming a definite amount of energy per unit area. The nucleation and motion of phase boundaries, including material stability aspects, was investigated by Dayal and Bhattacharya (2006).

In Sect. 2 of this paper, the fundamentals of the peridynamic theory are reviewed. In Sect. 3, the linearized equations are discussed that represent the superposition of a small displacement field on a large equilibrated deformation. In Sect. 4, the evolution of such a superposed displacement field containing a jump discontinuity is investigated, resulting in a material stability condition. The relationship between this condition and the wave speeds in the material is discussed in Sect. 5. A computational example illustrating the nucleation of a crack at a stress concentration in a plate is presented in Sect. 6.

\section{Basic equations of peridynamics}

In the peridynamic model, the strain energy density at a point $\mathbf{x}$ in an elastic body $\mathcal{B}$ is determined by the collective deformation of all the points within a predefined interaction distance of $\mathbf{x}$. This interaction distance is called the horizon and denoted $\delta$. The relative position vector in the reference configuration from $\mathbf{x}$ to any point $\mathbf{q}$ within the horizon is called a bond, denoted $\boldsymbol{\xi}=\mathbf{q}-\mathbf{x}$. 
The set of all bonds connected to $\mathbf{x}$ is called the family of $\mathbf{x}$ and denoted $\mathcal{H}$.

In the constitutive modeling of a peridynamic elastic material, the basic kinematical quantity that determines the strain energy density $W(\mathbf{x}, t)$ is called the deformation state at $\mathbf{x}$ and $t$, defined by

$\underline{\mathbf{Y}}[\mathbf{x}, t]\langle\xi\rangle=\mathbf{y}(\mathbf{x}+\boldsymbol{\xi}, t)-\mathbf{y}(\mathbf{x}, t)$

where $\mathbf{y}$ is the motion. The deformation state is a mapping that associates with any bond $\xi$ its image under the motion. The bond that $\underline{\mathbf{Y}}$ operates on is written in angle brackets, $\langle\boldsymbol{\xi}\rangle$.

The deformation state is an example of a vector state, which is simply a mapping from bonds to vectors in $\mathbb{R}^{3}$. The dot product of two vector states $\underline{\mathbf{A}}$ and $\underline{\mathbf{B}}$ is defined by

$$
\underline{\mathbf{A}} \bullet \underline{\mathbf{B}}=\int_{\mathcal{H}} \underline{\mathbf{A}}\langle\boldsymbol{\xi}\rangle \cdot \underline{\mathbf{B}}\langle\boldsymbol{\xi}\rangle d V_{\boldsymbol{\xi}}
$$

where the integrand is the usual scalar product in $\mathbb{R}^{3}$ of the two vectors $\underline{\mathbf{A}}\langle\boldsymbol{\xi}\rangle$ and $\underline{\mathbf{B}}\langle\boldsymbol{\xi}\rangle$. The norm of a vector state is defined by

$$
\|\underline{\mathbf{A}}\|=\sqrt{\underline{\mathbf{A}} \bullet \underline{\mathbf{A}}} \text {. }
$$

If $\Psi$ is a scalar valued function of a vector state $\underline{\mathbf{A}}$, then its Fréchet derivative $\nabla \Psi(\underline{\mathbf{A}})$, if it exists, is defined by

$$
\Psi(\underline{\mathbf{A}}+\underline{\mathbf{a}})=\Psi(\underline{\mathbf{A}})+\nabla \Psi(\underline{\mathbf{A}}) \bullet \underline{\mathbf{a}}+o(\| \underline{\mathbf{a}}||)
$$

for any vector state $\underline{\mathbf{a}}$.

A double state $\mathbb{D}$ is a mapping from pairs of bonds to second order tensors; thus the value of $\mathbb{D}\langle\boldsymbol{\xi}, \zeta\rangle$ is a second order tensor. The adjoint of a double state is defined by

$$
\underline{\mathbb{D}}^{\dagger}\langle\boldsymbol{\xi}, \zeta\rangle=\underline{\mathbb{D}}^{T}\langle\zeta, \xi\rangle
$$

for any bonds $\xi$ and $\zeta$, where the superscipt $T$ indicates the tensor transpose. The dot product of a double state $\underline{\mathbb{D}}$ with a vector state $\underline{\mathbf{A}}$ is a vector state defined by

$$
(\underline{\mathbb{D}} \bullet \underline{\mathbf{A}})\langle\boldsymbol{\xi}\rangle=\int_{\mathcal{H}} \underline{\mathbb{D}}\langle\boldsymbol{\xi}, \boldsymbol{\zeta}\rangle \underline{\mathbf{A}}\langle\boldsymbol{\zeta}\rangle d V_{\zeta}
$$

for any bond $\boldsymbol{\xi}$. If $\underline{\mathbf{S}}$ is a vector state valued function of a vector state, its Fréchet derivative $\nabla \underline{\mathbf{S}}(\underline{\mathbf{A}})$, if it exists, is defined by

$$
\underline{\mathbf{S}}(\underline{\mathbf{A}}+\underline{\mathbf{a}})=\underline{\mathbf{S}}(\underline{\mathbf{A}})+\nabla \underline{\mathbf{S}}(\underline{\mathbf{A}}) \bullet \underline{\mathbf{a}}+o(\|\underline{\mathbf{a}}\|)
$$

for any vector state $\underline{\mathbf{a}}$.

In the constitutive modeling of an elastic material, the strain energy density $W(\mathbf{x}, t)$ is, in general, determined by the collective deformation of all the bonds

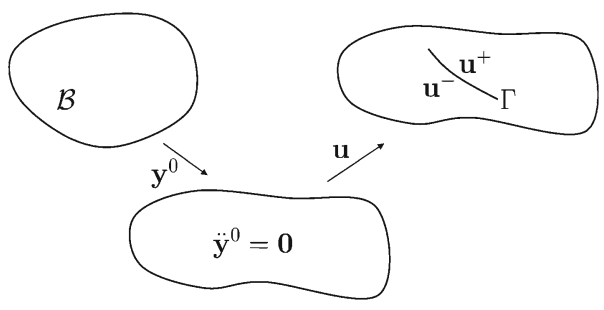

Fig. 1 An incremental displacement field $\mathbf{u}$ containing a jump across the surface $\Gamma$ is superposed on an equilibrated, possibly large deformation $\mathbf{y}^{0}$

connected to $\mathbf{x}$, not merely by the individual bonds independently of each other. It is this collective dependence that leads to the need for $W$ to be considered a function of $\underline{\mathbf{Y}}$. Thus, the constitutive model for an elastic material is written

$W(\mathbf{x}, t)=\hat{W}(\underline{\mathbf{Y}}[\mathbf{x}, t], \mathbf{x})$,

in which the explicit dependence of $\hat{W}$ on $\mathbf{x}$ reflects possible heterogeneity. The functional for total potential energy of a bounded body $\mathcal{B}$ in terms of the motion $\mathbf{y}$ is given by

$\Phi_{\mathbf{y}}=\int_{\mathcal{B}}(\hat{W}(\underline{\mathbf{Y}}[\mathbf{x}], \mathbf{x})-\mathbf{b} \cdot \mathbf{y}) d V_{\mathbf{x}}$,

where $\mathbf{b}$ is a prescribed body force density field. Deriving the Euler-Lagrange equation associated with stationary values of $\Phi_{\mathbf{y}}$, and applying d'Alembert's principle, leads to the peridynamic equation of motion:

$$
\begin{array}{r}
\rho(\mathbf{x}) \ddot{\mathbf{y}}(\mathbf{x}, t)=\int_{\mathcal{B}}\{\underline{\mathbf{T}}[\mathbf{x}, t]\langle\mathbf{q}-\mathbf{x}\rangle-\underline{\mathbf{T}}[\mathbf{q}, t] \\
\langle\mathbf{x}-\mathbf{q}\rangle\} d V_{\mathbf{q}}+\mathbf{b}(\mathbf{x}, t)
\end{array}
$$

where $\underline{\mathbf{T}}$ is the force state, a vector state defined by

$\underline{\mathbf{T}}[\mathbf{x}, t]=\underline{\hat{\mathbf{T}}}(\underline{\mathbf{Y}}[\mathbf{x}, t], \mathbf{x})=\nabla \hat{W}(\underline{\mathbf{Y}}[\mathbf{x}, t], \mathbf{x})$,

in which $\nabla$ denotes the Fréchet derivative with respect to $\underline{\mathbf{Y}}$. $\hat{\mathbf{T}}$ is the constitutive model that gives the force state as a function of the deformation state. The force state is the work conjugate of the deformation state:

$\hat{W}(\underline{\mathbf{Y}}+d \underline{\mathbf{Y}})-\hat{W}(\underline{\mathbf{Y}})=\underline{\mathbf{T}} \bullet d \underline{\mathbf{Y}}$.

\section{Linearized form of peridynamics}

Consider an equilibrated, continuous deformation $\mathbf{y}^{0}$ corresponding to time-independent external body force 
density field $\mathbf{b}$. Let $\mathbf{u}$ be a small variation in the deformation (Fig. 1). Linearizing the function $\hat{\mathbf{T}}$ near $\mathbf{y}^{0}$ leads to

$\underline{\mathbf{T}}=\underline{\mathbf{T}}^{0}+\underline{\mathbb{K}} \bullet \underline{\mathbf{U}}+o(\|\underline{\mathbf{U}}\|)$

where

$\underline{\mathbf{T}}^{0}[\mathbf{x}]=\underline{\hat{\mathbf{T}}}\left(\underline{\mathbf{Y}}^{0}[\mathbf{x}], \mathbf{x}\right), \quad \underline{\mathbf{U}}[\mathbf{x}]\langle\mathbf{p}-\mathbf{x}\rangle=\mathbf{u}(\mathbf{p})-\mathbf{u}(\mathbf{x})$

for all points $\mathbf{x}$ and $\mathbf{p}$ in $\mathcal{B} . \underline{\mathbb{K}}$ is a double state called the modulus state defined, through the second Fréchet derivative of the strain energy density function, by

$\underline{\mathbb{K}}[\mathbf{x}]=\nabla \nabla \hat{W}\left(\underline{\mathbf{Y}}^{0}[\mathbf{x}], \mathbf{x}\right)$.

The modulus state is analogous to the fourth order elasticity tensor in the standard theory, because it contains the material properties that give the internal forces within a body in terms of small changes in the deformation. From the properties of Fréchet derivatives, and from (6), it can be shown that $\underline{\mathbb{K}}$ is self-adjoint (Silling 2010):

$\underline{\mathbb{K}}^{\dagger}=\underline{\mathbb{K}}$.

Using (4), and neglecting terms of higher order than $\|\underline{\mathbf{U}}\|$, the equation of motion (2) becomes

$$
\begin{aligned}
\rho(\mathbf{x}) \ddot{\mathbf{u}}(\mathbf{x}, t)= & \int_{\mathcal{B}}\{(\underline{\mathbb{K}}[\mathbf{x}] \bullet \underline{\mathbf{U}}[\mathbf{x}])\langle\mathbf{p}-\mathbf{x}\rangle \\
& -(\underline{\mathbb{K}}[\mathbf{p}] \bullet \underline{\mathbf{U}}[\mathbf{p}])\langle\mathbf{x}-\mathbf{p}\rangle\} d V_{\mathbf{p}}+\mathbf{b}(\mathbf{x}, t) .
\end{aligned}
$$

Writing this out in terms of the displacements using (5) yields

$$
\begin{aligned}
& \rho(\mathbf{x}) \ddot{\mathbf{u}}(\mathbf{x}, t) \\
& =\int_{\mathcal{B}} \mathbf{C}_{0}(\mathbf{x}, \mathbf{q}) \mathbf{u}(\mathbf{q}, t) d V_{\mathbf{q}}-\mathbf{P}_{0}(\mathbf{x}) \mathbf{u}(\mathbf{x}, t)
\end{aligned}
$$

where $\mathbf{C}_{0}$ is the tensor valued function defined by

$$
\begin{aligned}
& \mathbf{C}_{0}(\mathbf{x}, \mathbf{q}) \\
& =\int_{\mathcal{B}}(\underline{\mathbb{K}}[\mathbf{x}]\langle\mathbf{p}-\mathbf{x}, \mathbf{q}-\mathbf{x}\rangle-\underline{\mathbb{K}}[\mathbf{p}]\langle\mathbf{x}-\mathbf{p}, \mathbf{q}-\mathbf{p}\rangle \\
& \quad+\underline{\mathbb{K}}[\mathbf{q}]\langle\mathbf{x}-\mathbf{q}, \mathbf{p}-\mathbf{q}\rangle) d V_{\mathbf{p}}
\end{aligned}
$$

and where $\mathbf{P}_{0}$ is the tensor field defined by

$\mathbf{P}_{0}(\mathbf{x})=\int_{\mathcal{B}} \int_{\mathcal{B}} \underline{\mathbb{K}}[\mathbf{x}]\langle\mathbf{p}-\mathbf{x}, \mathbf{q}-\mathbf{x}\rangle d V_{\mathbf{q}} d V_{\mathbf{p}}$

By integrating (9) with respect to $\mathbf{q}$, it follows that

$\mathbf{P}_{0}(\mathbf{x})=\int_{\mathcal{B}} \mathbf{C}_{0}(\mathbf{x}, \mathbf{q}) d V_{\mathbf{q}}$

In practice, the expressions for $\mathbf{C}_{0}$ in many materials of interest contain Dirac delta functions centered at $\mathbf{x}$.
It is convenient to move this term outside the integral in the equation of motion (8) by rewriting it as

$$
\begin{gathered}
\rho(\mathbf{x}) \ddot{\mathbf{u}}(\mathbf{x}, t)=\int_{\mathcal{B}} \mathbf{C}(\mathbf{x}, \mathbf{q}) \mathbf{u}(\mathbf{q}, t) d V_{\mathbf{q}}-\mathbf{P}(\mathbf{x}) \mathbf{u}(\mathbf{x}, t) \\
+\mathbf{b}(\mathbf{x}, t),
\end{gathered}
$$

for all $\mathbf{x}$ and $t$, where

$$
\begin{aligned}
\mathbf{C}(\mathbf{x}, \mathbf{q}) & =\mathbf{C}_{0}(\mathbf{x}, \mathbf{q})+\lambda(\mathbf{x}) \Delta(\mathbf{q}-\mathbf{x}), \\
\mathbf{P}(\mathbf{x}) & =\mathbf{P}_{0}(\mathbf{x})+\lambda(\mathbf{x})=\int_{\mathcal{B}} \mathbf{C}(\mathbf{x}, \mathbf{q}) d V_{\mathbf{q}}, \\
\lambda(\mathbf{x}) & =-\lim _{\epsilon \rightarrow 0} \int_{\mathcal{S}_{\epsilon}} \mathbf{C}_{0}(\mathbf{x}, \mathbf{q}) d V_{\mathbf{q}},
\end{aligned}
$$

where $\mathcal{S}_{\epsilon}$ is the interior of a sphere of radius $\epsilon$ centered at $\mathbf{x}$, and $\Delta$ is the Dirac delta function in $\mathbb{R}^{3}$. For an elastic material, it can be shown (Silling 2010) that C and $\mathbf{P}$ have the following symmetries:

$\mathbf{C}^{T}(\mathbf{x}, \mathbf{q})=\mathbf{C}(\mathbf{q}, \mathbf{x}), \quad \mathbf{P}^{T}(\mathbf{x})=\mathbf{P}(\mathbf{x})$.

As discussed in Silling (2010), the condition for linearization of the peridynamic equations is

$\min _{\mathbf{q} \in \mathcal{H}}|\mathbf{u}(\mathbf{q})-\mathbf{u}(\mathbf{x})|<<\delta \quad \forall \mathbf{x} \in \mathcal{B}$.

This is a weaker condition than the corresponding requirement in the classical linear theory of elasticity, $|\operatorname{grad} \mathbf{u}(\mathbf{x})|<<1 \quad \forall \mathbf{x} \in \mathcal{B}$.

The weaker requirement in the linear peridynamic theory permits us to consider perturbations containing jump discontinuities in $\mathbf{u}$, a property that is used in the next section. The linearized equation of motion (11) is essentially the same as the fundamental equation of motion in Kunin's nonlocal theory (Kunin 1983), but with the additional constitutive structure of the peridynamic model.

\section{Perturbation by a jump}

Now consider a piecewise-continuous variation $\mathbf{u}$ that contains a small step discontinuity across a surface $\Gamma$. Assume that $\Gamma$ divides $\mathcal{B}$ into two open sets $\mathcal{R}^{+}$and $\mathcal{R}^{-}$. Let $\mathbf{C}$ be continuous on $\mathcal{B} \times \mathcal{B}$. Let the jumps in displacement and acceleration at some $\mathbf{x} \in \Gamma$ be denoted

$[[\mathbf{u}]]=\mathbf{u}\left(\mathbf{x}^{+}\right)-\mathbf{u}\left(\mathbf{x}^{-}\right), \quad[[\ddot{\mathbf{u}}]]=\ddot{\mathbf{u}}\left(\mathbf{x}^{+}\right)-\ddot{\mathbf{u}}\left(\mathbf{x}^{-}\right)$ where $\mathbf{x}^{-} \in \mathcal{R}^{-}$and $\mathbf{x}^{+} \in \mathcal{R}^{+}$represent points arbitrarily close to $\mathbf{x}$. Then from (11),

$\rho(\mathbf{x})[[\ddot{\mathbf{u}}]]=\int_{\mathcal{B}}[[\mathbf{C}(\mathbf{x}, \mathbf{q})]] \mathbf{u}(\mathbf{q}) d V_{\mathbf{q}}-[[\mathbf{P u}]]$. 
Since $\mathbf{C}$, and therefore $\mathbf{P}$, are continuous, it follows that

$[[\mathbf{C}]]=\mathbf{0}$ and $[[\mathbf{P}]]=\mathbf{0}$,

therefore

$\rho(\mathbf{x})[[\ddot{\mathbf{u}}]]=-\mathbf{P}(\mathbf{x})[[\mathbf{u}]]$.

The small discontinuity at $\mathbf{x} \in \Gamma$ introduced by the variation is considered unstable if it gets larger with time. This means it is unstable if

$[[\ddot{\mathbf{u}}]] \cdot[[\mathbf{u}]]>0$.

Taking the scalar product of $[[\mathbf{u}]]$ with both sides of (15), and using (16), the conclusion is that instability occurs if

$$
(\mathbf{P}(\mathbf{x})[[\mathbf{u}]]) \cdot[[\mathbf{u}]]<0 .
$$

If there is some [[u] ] such that (17) holds at some $\mathbf{x}$, then the material is unstable with respect to amplification of a discontinuity. ${ }^{1}$ Since $\mathbf{P}(\mathbf{x})$ is symmetric, it has three real eigenvalues, which will be denoted $\left\{P_{1}, P_{2}, P_{3}\right\}$. (17) implies that if $\mathbf{P}(\mathbf{x})$ has a negative eigenvalue, then the material is unstable with respect to amplification of the jump. The condition for crack nucleation is then

$Z(\mathbf{x})<0$,

where

$Z(\mathbf{x})=\min \left\{P_{1}, P_{2}, P_{3}\right\}$.

$Z(\mathbf{x})$ is called the stability index.

If $\mathbf{P}$ is positive definite on $\mathcal{B}$, then in the terminology of Silling (2000), single-point stability holds. In this case, then clearly (18) cannot hold, since $\mathbf{P}$ has no negative eigenvalues. In Silling (2000), it is shown that single-point stability is a necessary but not sufficient condition for local minimization of potential energy. Therefore, it follows that if the nucleation condition (18) is met, then the configuration cannot be a local minimizer of potential energy.

A result closely related to (18) was also obtained in Weckner and Abeyaratne (2005) in a more restrictive setting. In this reference, it was shown that in a homogeneous, bond-based, linear microelastic bar, jumps in an initial displacement field grow exponentially over time if

$$
\int_{-\infty}^{\infty} C(\xi) d \xi<0
$$

\footnotetext{
${ }^{1}$ The word "amplification" is used instead of "growth" here to avoid confusion with advance of a crack tip.
}

where $C$ is the micromodulus function. This integral is the specialization of $\mathbf{P}$ in (13) to a linear, homogeneous bar.

To interpret $\mathbf{P}$ mechanically, note that from (11), it follows that $-\mathbf{P}(\mathbf{x}) \mathbf{e}$ is the restoring force density experienced by $\mathbf{x}$ if it is displaced by a vector $\mathbf{e}$, holding all other points in $\mathcal{B}$ fixed. In the next section, a connection between $\mathbf{P}$ and the stable propagation of certain types of plane waves is demonstrated.

\section{Relation to wave speeds}

An alternate interpretation of (18) may be obtained by considering plane waves in the interior of a large, homogeneous, homogeneously deformed body. In this case, $\mathbf{P}$ is independent of position. Since $\mathbf{C}$ is also independent of position, we write $\mathbf{C}(\boldsymbol{\xi})$. The condition for propagation of a plane wave may be obtained by substituting the assumed form of the displacement field

$\mathbf{u}(\mathbf{x}, t)=\mathbf{a} e^{i(\kappa \mathbf{x} \cdot \mathbf{n}-\omega t)}$

into (11), where $i=\sqrt{-1}$, $\mathbf{a}$ is a unit vector in the direction of particle motion, $\mathbf{n}$ is the unit vector in the direction of propagation of the wave, $\kappa$ is the wave number, and $\omega$ is the angular frequency. The result is that waves can exist if there exist $\mathbf{a}, \mathbf{n}, \kappa$, and $\omega(\kappa, \mathbf{n})$ such that

$\rho \omega^{2}(\kappa, \mathbf{n}) \mathbf{a}=\left(\mathbf{P}-\int_{\mathcal{H}} \mathbf{C}(\boldsymbol{\xi}) \cos (\kappa \xi \cdot \mathbf{n}) d V_{\xi}\right) \mathbf{a}$.

The quantity in parentheses is a tensor. The dispersion relation for the waves is obtained by evaluating the eigenvalue $\omega$ of this tensor for a given $\kappa$ and $\mathbf{n}$. The eigenvectors a give the direction of particle motion.

Now consider the limit $\kappa \rightarrow \infty$ in (20). Because $\mathbf{C}$ is bounded and continuous, the integral vanishes in this limit, for any choice of $\mathbf{n}$. Therefore, the eigenvalue equation (20) can be written as

$\rho \omega^{2}(\infty, \mathbf{n}) \mathbf{a}=\mathbf{P a}$.

Taking the scalar product of both sides of this equation with the unit vector $\mathbf{a}$,

$\rho \omega^{2}(\infty, \mathbf{n})=\mathbf{a} \cdot \mathbf{P a}$.

Comparing this result with (17) and (18), it follows that the condition for nucleation of discontinuities is met if and only if the frequency of plane waves in the limit of zero wavelength is imaginary. 
Note that it is possible for waves with finite wave number to be unstable even if this limiting frequency is real. In other words, long waves might be unstable while short waves are stable. This is in contrast to the standard theory, in which the stability of plane waves is independent of the wavelength.

The interpretation of the crack nucleation condition (18) given in this section in terms of imaginary frequencies of very short waves should not be taken too literally, because this interpretation assumes a homogeneously deformed, homogeneous body. In contrast, (18) makes no such assumptions, and is therefore more general.

\section{Example}

This computational example illustrates the nucleation of a crack at a point at which the condition (18) is first met as the body is loaded. The body is a nonlinearly elastic plate containing a hole and stretched from the ends. The constitutive model is a nonlinear peridynamic elastic model of the following form:

$\underline{\mathbf{T}}\langle\boldsymbol{\xi}\rangle=\frac{1}{2} f(\eta, \xi) \mathbf{M}, \quad \mathbf{M}=\frac{\underline{\mathbf{Y}}\langle\boldsymbol{\xi}\rangle}{|\underline{\mathbf{Y}}\langle\boldsymbol{\xi}\rangle|}$,

for any bond $\boldsymbol{\xi}$, where $f$ is a function and the bond extension $\eta$ and bond length $\xi$ are given by

$\eta=|\underline{\mathbf{Y}}\langle\boldsymbol{\xi}\rangle|-|\boldsymbol{\xi}|, \quad \xi=|\boldsymbol{\xi}|$.

The unit vector $\mathbf{M}$ is in the direction of the deformed bond. The scalar valued function $f$ is the bond force density, which has dimensions of force/volume ${ }^{2}$. The direction of the force state $\underline{\mathbf{T}}\langle\boldsymbol{\xi}\rangle$ is parallel to the deformed bond vector $\underline{\mathbf{Y}}\langle\boldsymbol{\xi}\rangle$. For the particular material in this example, $f$ is given by

$$
\begin{aligned}
& f(\eta, \xi) \\
& \quad=\left\{\begin{array}{ll}
c s & \text { if } 0 \leq s \leq s_{1} \\
c s_{1}-c^{\prime}\left(s-s_{1}\right) & \text { if } s_{1} \leq s<s_{0} \\
0 & \text { if } s_{0}<s,
\end{array} \quad s=\frac{\eta}{\xi}\right.
\end{aligned}
$$

where $c, c^{\prime}, s_{1}$, and $s_{0}$ are constants related by $c^{\prime}=\frac{c s_{1}}{s_{0}-s_{1}}, \quad 0<s_{1}<s_{0}, \quad c>0$.

(See Fig. 2). The scalar $s$ is the bond strain, i.e., change in bond length divided by reference bond length. Evaluating the Fréchet derivative of (22), one easily finds that

$$
\begin{aligned}
& \underline{\mathbb{K}}\langle\boldsymbol{\xi}, \zeta\rangle \\
& =\frac{1}{2}\left(\frac{\mathbf{1}-\mathbf{M} \otimes \mathbf{M}}{|\underline{\mathbf{Y}}\langle\boldsymbol{\xi}\rangle|} f+(\mathbf{M} \otimes \mathbf{M}) \frac{\partial f}{\partial \eta}\right) \Delta(\boldsymbol{\xi}-\boldsymbol{\zeta})
\end{aligned}
$$

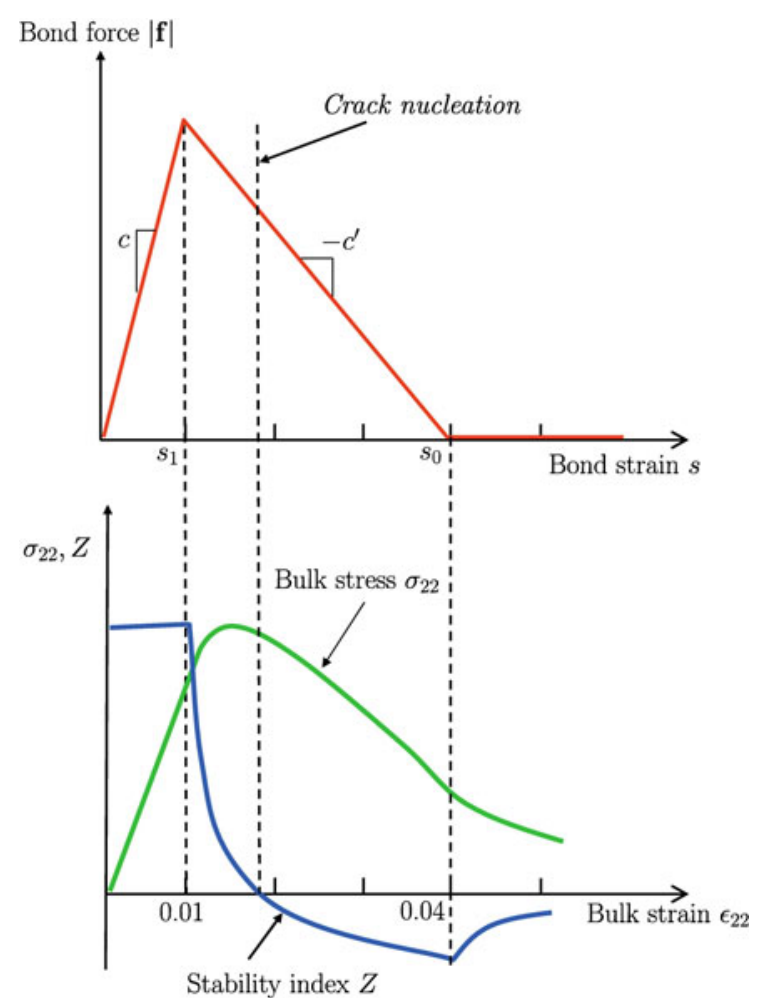

Fig. 2 Properties of a plate composed of the material in the example problem under uniaxial strain. Top: bond force as a function of bond strain. Bottom: stress-strain curves in a plate under homogeneous, uniaxial strain. The peak stress occurs before the crack nucleation condition is met

where $\Delta$ denotes the Dirac delta function in three dimensions.

The top curve in Fig. 2 shows the bond force as a function of bond strain for the material. Also shown are the normal stress in the vertical direction and the stability index $Z$ as a function of bulk strain, both assuming homogeneous deformation of a homogeneous plate. The stability index starts dropping when some bonds pass the peak in the curve of bond force vs. bond strain. As the plate is stretched more, the stability index eventually becomes negative, thus satisfying the condition (18) for nucleation of a discontinuity. (This condition occurs at a strain greater than the peak in the bulk stressstrain curve.)

The geometry of the plate is shown in Fig. 3. Figure 4 shows contours of $u_{2}$ and $Z$ near the stress concentration for three values of global strain, defined by $\varepsilon=\Delta \ell / \ell$. The displacement field is computed with the Emu peridynamic computer code (Silling and Askari 2005). A small crack appears at a global strain 


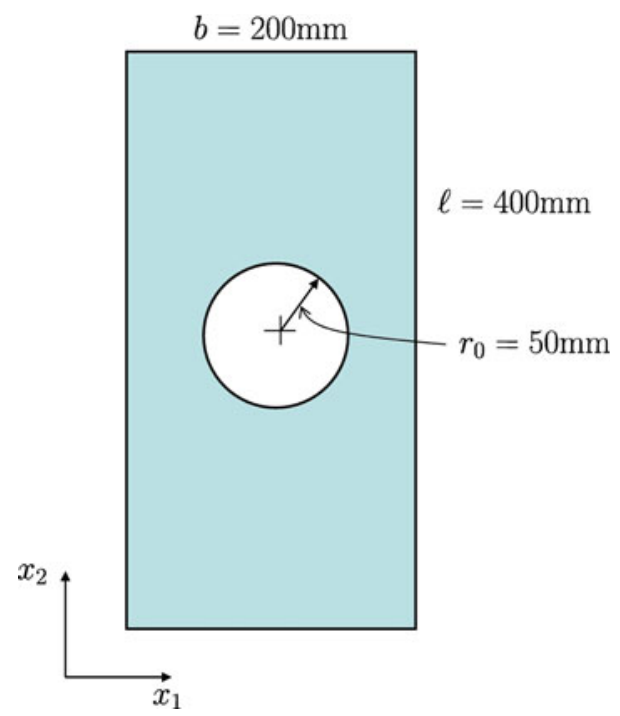

Fig. 3 Geometry of the plate in Example 1

of approximately $\varepsilon=0.0049$, by which time $Z$ has become small at the stress concentration. The crack immediately grows dynamically toward the outer free edges. The contours in the plot on the lower right show that $Z$ becomes strongly negative ahead of a dynamically growing crack tip. Figure 5 shows the values of $Z$ at the point $\mathbf{x}_{0}$, which is located $54 \mathrm{~mm}$ from the center of the hole as a function of global strain. The figure also shows the crack tip position $a$ as a function of global strain. The crack first appears at $a \approx 54 \mathrm{~mm}$, at the time when $Z$ at that location first becomes negative. As soon as the crack nucleates, $Z$ at this location becomes positive again, because the material is no longer unstable there.

\section{Discussion}

Even though the peridynamic theory is a nonlocal theory, the crack nucleation condition (18) is a local condition, because it involves only the value of the tensor field $\mathbf{P}$ at $\mathbf{x}$. This $\mathbf{P}(\mathbf{x})$ can be evaluated anywhere in the body in terms of the incremental properties of the constitutive model evaluated following the large equilibrated deformation $\mathbf{y}$.

The crack nucleation condition (18) is not related to the maximum of any bulk stress-strain curve for a homogeneous body or to any tangent modulus. To see this, consider the form of (19) for a one-dimensional homogeneous bar:

$Z=\int_{-\delta}^{\delta} C(\xi) d \xi$
Fig. 4 Contours of vertical displacement $u_{2}$ (left column) and stability index $Z$ (right column) at three times. The global stretch of the plate $\varepsilon=\Delta \ell / \ell$ at these times takes on the values $0.0047,0.0049$, and 0.0061 . Crack nucleation occurs at approximately $\varepsilon=0.0049$. The stability index first becomes negative near this time and becomes more strongly negative in front of the growing crack tip

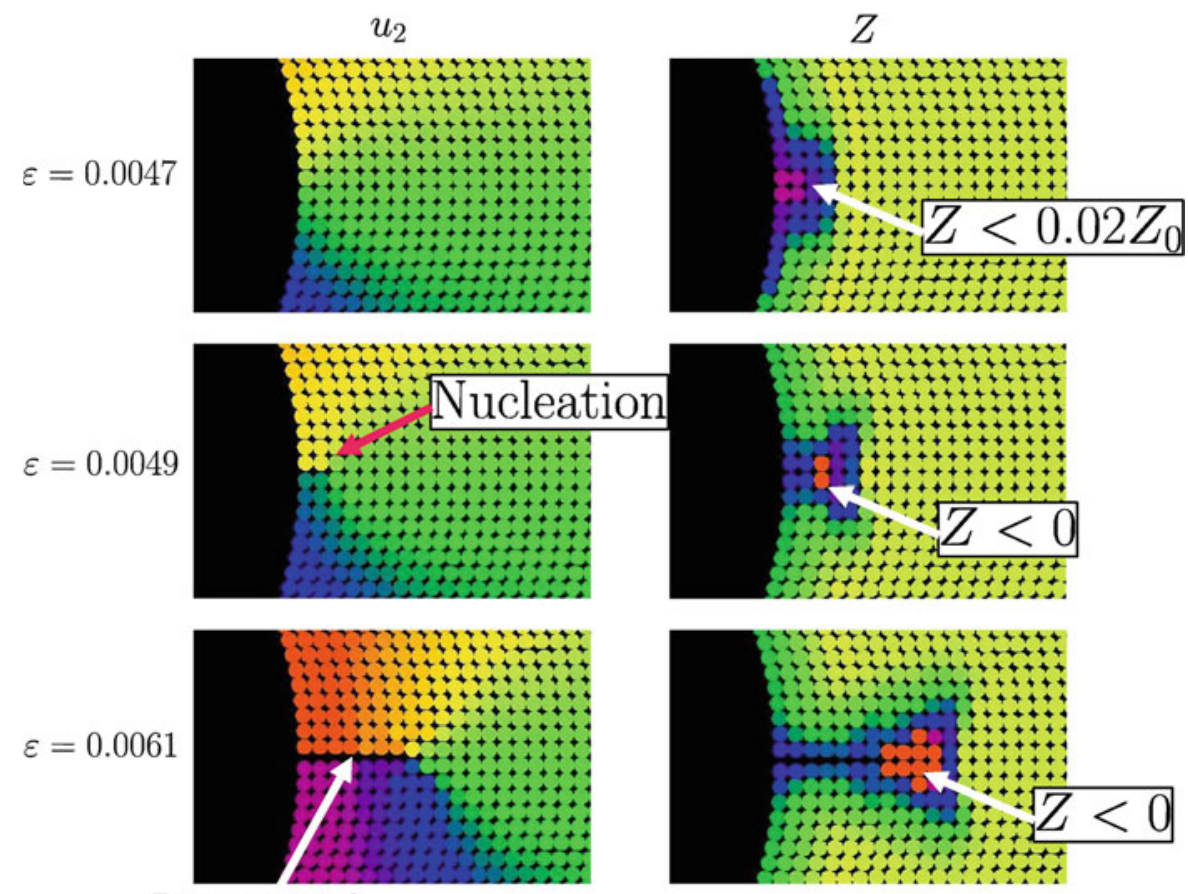

Dynamic crack 
Fig. 5 Time histories of stability index $Z$ (top) and crack tip position $a$ (bottom) at the point $\mathbf{x}_{0}$, located at radius $r_{0}=0.054 \mathrm{~mm}$. Nucleation occurs at near this point as $Z$ becomes negative
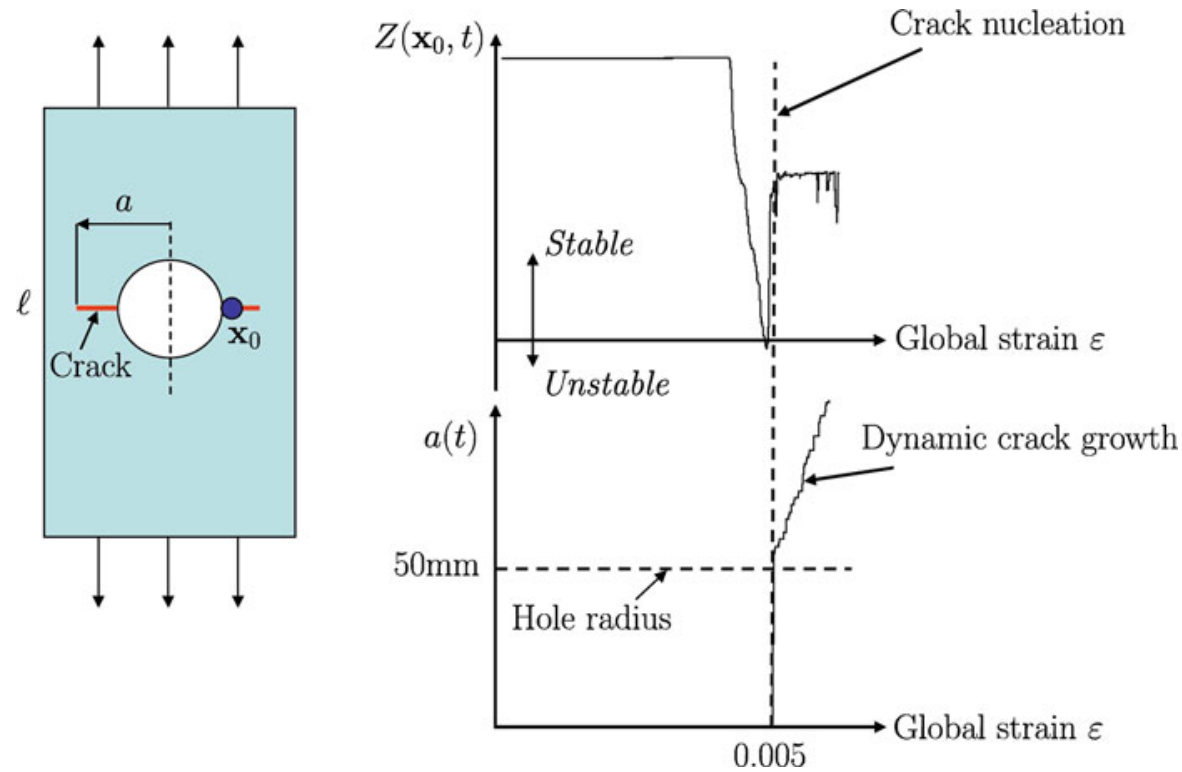

The eigenvector of $\mathbf{P}(\mathbf{x})$ that first becomes negative as the body is deformed, resulting in the condition (18) first being met, gives the direction of the displacement jump $[[\mathbf{u}]]$. However, we have not been able to obtain any information about the orientation of a crack that is nucleated. Another currently unresolved issue is the status of bodies in which $Z=0$. It is not known whether this condition, as opposed to $Z<0$, represents a type of neutral stability with respect to the formation of discontinuities, or whether closer examination would reveal a different condition for nucleation in this case.

As demonstrated computationally in the example in Sect. 6, there is a subregion ahead of a dynamically growing crack within which $Z$ is strongly negative. It seems possible that future investigation will establish a link between the values of $Z$ ahead of such a crack, the crack growth velocity, and [[ن̈]] near the crack tip.

Acknowledgments Sandia is a multiprogram laboratory operated by Sandia Corporation, a Lockheed Martin Company, for the United States Department of Energy's National Nuclear Security Administration under contract DE-AC04-94AL85000.

\section{References}

Agwai A, Guven I, Madenci E (2008) Peridynamic theory for failure prediction in multilayer thin-film structures of electronic packages. In: 2008 electronic components and technology conference. IEEE, pp 1614-1619

Agwai A, Guven I, Madenci E (2008) Peridynamic theory for impact damage prediction in electronic packages due to 
drop. In: 2008 electronic components and technology conference. IEEE, pp 1048-1053

Agwai A, Guven I, Madenci E (2009) Damage prediction for electronic package drop test using finite element and peridynamic theory. In: 2008 electronic components and technology conference. IEEE, pp 565-569

Askari E, Xu J, Silling S (2006) Peridynamic analysis of damage and failure in composites. In: 44th AIAA aerospace sciences meeting and exhibit, Reno, NV, AIAA2006-88

Bazant ZP, Belytschko T (1985) Wave propagation in a strainsoftening bar: exact solution. J Eng Mech (ASCE) 111: 381-389

Belytschko T, Chen H, Xu J, Zi G (2003) Dynamic crack propagation based on loss of hyperbolicity and a new discontinuous enrichment. Int J Numer Methods Eng 58:1873-1905

Bobaru F (2007) Influence of van der Waals forces on increasing the strength and toughness in dynamic fracture of nanofibre networks: a peridynamic approach. Model Simul Mater Sci Eng 15:397-417

Colavito KW, Kilic B, Celik E, Madenci E, Askari E, Silling S (2007) Effect of void content on stiffness and strength of composites by a peridynamic analysis and static indentation test. In: 48th AIAA/ASME/ASCE/AHS/ASC structures, structural dynamics, and materials conference, Honolulu, HI, AIAA2007-2251

Dayal K, Bhattacharya K (2006) Kinetics of phase transformations in the peridynamic formulation of continuum mechanics. J Mech Phys Solids 54:1811-1842

Gerstle W, Sau N, Silling S (2007) Peridynamic modeling of concrete structures. Nucl Eng Des 237:1250-1258

Hill R (1962) Acceleration waves in solids. J Mech Phys Solids 10:1-16

Kilic B, Madenci E (2009) Prediction of crack paths in a quenched glass plate by using peridynamic theory. Int $\mathrm{J}$ Fract 156:165-177

Kilic B, Agwai A, Madenci E (2009) Peridynamic theory for progressive damage prediction in center-cracked composite laminates. Compos Struct 90:141-151

Klein P, Gao H (1998) Crack nucleation and growth as strain localization in a virtual-bond continuum. Eng Fract Mech 61:21-48
Knowles JK, Sternberg E (1975) On the ellipticity of the equations of nonlinear elastostatics for a special material. J Elast $5: 341-361$

Knowles JK, Sternberg E (1978) On the failure of ellipticity and the emergence of discontinuous deformation gradients in plane finite elastostatics. J Elast 8:329-379

Kunin IA (1983) Elastic media with microstructure II: threedimensional models. Springer, Berlin

Leroy Y, Ortiz M (1989) Finite element analysis of strain localization in frictional materials. Int J Numer Anal Methods Geomech 13:53-74

Rudnicki JW, Rice JR (1975) Conditions for the localization of deformation in pressure-sensitive dilatant materials. J Mech Phys Solids 23:371-394

Silling SA (2000) Reformulation of elasticity theory for discontinuities and long-range forces. J Mech Phys Solids 48:175209

Silling SA (2003) Dynamic fracture modeling with a meshfree peridynamic code. In: Bathe KJ (ed) Computational fluid and solid mechanics. Elsevier, Amsterdam, pp 641-644

Silling SA, Askari E (2005) A meshfree method based on the peridynamic model of solid mechanics. Comput Struct 83:1526-1535

Silling SA, Bobaru F (2005) Peridynamic modeling of membranes and fibers. Int J Non-Linear Mech 40:395-409

Silling SA, Epton M, Weckner O, Xu J, Askari E (2007) Peridynamic states and constitutive modeling. J Elast 88:151-184

Silling SA (2010) Linearized theory of peridynamic states. J. Elast. doi:10.1007/s10659-009-9234-0

Xu J, Askari A, Weckner O, Razi H, Silling S (2007) Damage and failure analysis of composite laminates under biaxial loads. In: 48th AIAA/ASME/ASCE/AHS/ASC structures, structural dynamics, and materials conference, Honolulu, HI, AIAA2007-2315

Xu J, Askari A, Weckner O, Silling S (2008) Peridynamic analysis of impact damage in composite laminates. J Aerosp Eng 21:187-194

Weckner O, Abeyaratne R (2005) The effect of long-range forces on the dynamics of a bar. J Mech Phys Solids 53:705-728 